\title{
Investigation of transferred-electron oscillations in diamond
}

\author{
N. Suntornwipat, S. Majdi, M. Gabrysch, and J. Isberg ${ }^{\text {a) }}$ \\ Division for Electricity, Uppsala University, Box 534, S-751 21 Uppsala, Sweden
}

(Received 24 February 2016; accepted 14 May 2016; published online 25 May 2016; publisher error corrected 27 May 2016)

\begin{abstract}
The recent discovery of Negative Differential Mobility (NDM) in intrinsic single-crystalline diamond enables the development of devices for high frequency applications. The TransferredElectron Oscillator (TEO) is one example of such devices that uses the benefit of NDM to generate continuous oscillations. This paper presents theoretical investigations of a diamond TEO in the temperature range of 110 to $140 \mathrm{~K}$ where NDM has been observed. Our simulations map out the parameter space in which transferred-electron oscillations are expected to occur for a specific device geometry. The results are promising and indicate that it is possible to fabricate diamond based TEO devices. Published by AIP Publishing. [http://dx.doi.org/10.1063/1.4952766]
\end{abstract}

The carrier drift velocity in semiconductors is normally proportional to the electric field at low fields and saturates at high fields; nonetheless, Gunn observed that the velocity could behave in a different way. He discovered that at high fields, the electron drift velocity in Indium Phosphide and Gallium Arsenide decreased with an increase in the electric field. ${ }^{1,2}$ This was later called Negative Differential Mobility (NDM) or Negative Differential Resistance (NDR). ${ }^{3}$ The phenomenon generally appears in direct bandgap III-V and II-VI semiconductor materials with non-equivalent valleys, such as GaAs, ${ }^{1} \mathrm{InP},{ }^{1} \mathrm{InAs},{ }^{4} \mathrm{ZnSe},{ }^{5}$ and $\mathrm{CdTe} .{ }^{5-8}$ When increasing the applied electric field, electrons with a lower effective mass at the central conduction band valley are heated up and transferred into satellite valleys where they have a higher effective mass, thus decreasing the drift velocity and causing NDM to occur. This effect is not expected to occur in indirect bandgap materials but has previously been reported for silicon at a temperature below $45 \mathrm{~K} .^{9}$ Furthermore, the phenomenon has been observed in intrinsic Single-Crystalline Chemical Vapor Deposition (SC-CVD) diamond. ${ }^{10}$ With an applied electric field of 300 to $600 \mathrm{~V} / \mathrm{cm}$ in the [100] direction, the NDM effect was observed in a temperature range of 110 to $140 \mathrm{~K}$. Since diamond is an indirect bandgap material, this effect occurs in a somewhat different way. ${ }^{10}$

Diamond has six equivalent conduction band valleys oriented along the $\{100\}$ directions with a uniquely low scattering rate between these valleys. ${ }^{11,12}$ Short wavelength lattice vibrations are required for intervalley scattering but these are, in diamond, absent at low temperatures due to the rigid lattice. Electrons in these valleys have a different longitudinal $\left(m_{l}\right)$ and transversal effective mass $\left(m_{t}\right)$, which cause a strong anisotropy in the transport properties of the polarized electrons. If an electric field is applied in the [100] direction, two valleys are aligned in parallel (on the (100) axis) and four valleys orthogonal to the field (on the (010) and (001) axes). The electrons in the valleys on the (100) axis respond to the electric field with the longitudinal effective mass $\left(m_{l}=1.15 m_{0}\right)$, and

\footnotetext{
${ }^{\text {a) }}$ Author to whom correspondence should be addressed. Electronic mail: jan.isberg@angstrom.uu.se
}

electrons in the valleys on the (010) and (001) axes respond with the transversal effective mass $\left(m_{t}=0.22 m_{0}\right) \cdot{ }^{13}$ At low electric fields, the electron drift velocity is proportional to the field. By increasing the electric field, the electrons in the orthogonal valleys are heated up more than the electrons in the parallel valleys due to the difference in their effective masses $\left(m_{t}<m_{l}\right)$. The electrons from the orthogonal valleys reach the energy threshold for longitudinal acoustic (LA) phonon emission of $120 \mathrm{meV}$ (Ref. 14) first, which leads to a strong repopulation of conduction band electrons from the orthogonal to the parallel valleys. With an even higher electric field, the electrons will re-scatter into the orthogonal valleys whenever electrons in the parallel valleys reach a sufficient energy. This is shown in Fig. 1. If this repopulation is strong enough, the NDM effect can be observed. Electric current instabilities can then build up which give rise to oscillations called transferred-electron oscillations.

The Transferred-Electron Device (TED) or Gunn diode is one type of negative resistance oscillator which uses NDM to generate continuous oscillations. It is an important device in microwave applications up to $150 \mathrm{GHz}$ and is mainly used for microwave generation as a local oscillator or as a power amplifier. By applying an electric field within the region giving the negative resistance, the internal space charge

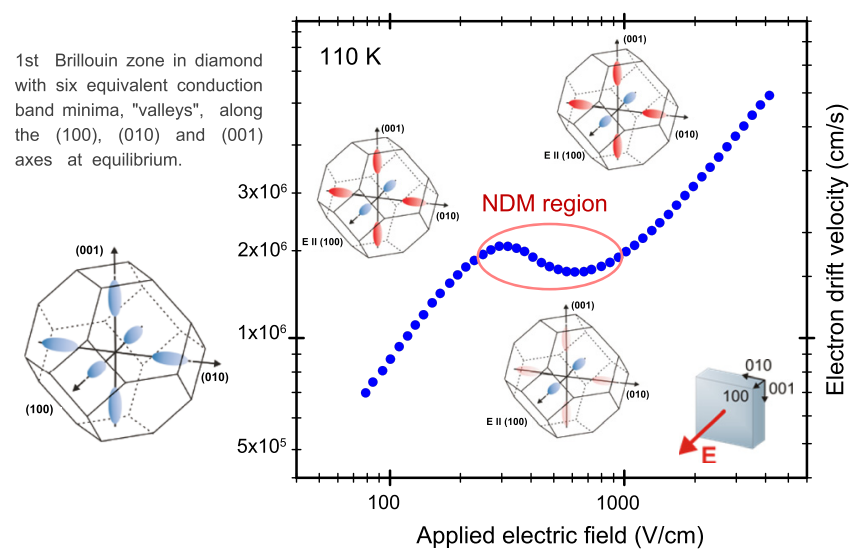

FIG. 1. Electron drift velocity as a function of the electric field for a SCCVD diamond sample at $110 \mathrm{~K}$. The number of electrons in the orthogonal and parallel valleys changes due to the scattering-induced repopulation. 
distribution and the internal electrical field distribution become unstable which makes the TED unique compared to other devices based on NDM, such as the tunnel diode and the impact ionization avalanche transit time (IMPATT) diode. ${ }^{15,16}$ Even though the efficiency and the output power of TEDs are generally lower than that of IMPATT diodes, TEDs typically generate less noise, operate at lower voltages, and are somewhat less complicated to design. It is not unlikely that TEDs will become important in solid-state microwave sources used in detection systems, remote controls, and microwave test instruments. ${ }^{16}$ In this paper, we investigate the theory of transferred-electron oscillations in diamond using a computational approach to map out parameters for which oscillations can be expected to occur in a proposed experiment.

One and two dimensional models of a TransferredElectron Oscillator (TEO) have been created in COMSOL Multiphysics and analyzed using its Finite Element Method (FEM) solver. A DC bias voltage was connected to a TED, in this case diamond, in series with a resonant circuit. This can be seen in Fig. 2(a). The TED model is based on SC diamond using Maxwell's displacement current and driftdiffusion equations at location $\boldsymbol{x}$ and time $t$, where the total current density can be found from

$$
\begin{aligned}
\boldsymbol{J}_{t o t}(\boldsymbol{x}, t) & =\boldsymbol{J}_{n}(\boldsymbol{x}, t)+\boldsymbol{J}_{p}(\boldsymbol{x}, t)-\varepsilon_{0} \varepsilon_{r} \frac{\partial}{\partial t} \nabla \Phi, \\
\boldsymbol{J}_{n}(\boldsymbol{x}, t) & =-q n \mu_{n}(E) \nabla \Phi+k T \mu_{n}(E) \nabla n, \\
\boldsymbol{J}_{p}(\boldsymbol{x}, t) & =-q p \mu_{p} \nabla \Phi-k T \mu_{p} \nabla p,
\end{aligned}
$$

where $\boldsymbol{J}_{t o t}(\boldsymbol{x}, t), \boldsymbol{J}_{n}(\boldsymbol{x}, t)$, and $\boldsymbol{J}_{p}(\boldsymbol{x}, t)$ are the total, electron, and hole current density; $\varepsilon_{r}$ and $\varepsilon_{0}$ are the relative permittivity of diamond and the vacuum permittivity; $\Phi$ is the electrostatic potential; $q$ is the elementary charge; $T$ is the lattice temperature; $k$ is the Boltzmann constant; $\mu_{p}$ is the hole mobility; $\mu_{n}(E)$ is the electron mobility as a function of the electric field; $n(\boldsymbol{x}, t)$ and $p(\boldsymbol{x}, t)$ are the electron and hole concentrations, respectively. The electron and hole concentrations can be calculated using Poisson's equation and the continuity equations

$$
\nabla^{2} \Phi=\frac{q}{\varepsilon_{0} \varepsilon_{r}}(n-p),
$$

$$
\begin{gathered}
\frac{\partial n}{\partial t}+\nabla\left(n \mu_{n}(E) \nabla \Phi-\frac{k T}{q} \mu_{n}(E) \nabla n\right)=g_{n}-R_{S R H}, \\
\frac{\partial p}{\partial t}+\nabla\left(-p \mu_{p} \nabla \Phi-\frac{k T}{q} \mu_{p} \nabla p\right)=g_{p}-R_{S R H}
\end{gathered}
$$

where $g_{n}$ and $g_{p}$ are the electron and hole generation rate and $R_{S R H}$ is the Shockley-Read-Hall (SRH) recombination rate. The SRH recombination rate is given by

$$
R_{S R H}=\frac{n p}{n \tau_{n}+p \tau_{p}},
$$

where $\tau_{n}$ and $\tau_{p}$ are the electron and hole life time, respectively. The simulations are based on previous results where we have observed the NDM effect in a temperature range of 110 to $140 \mathrm{~K}$ using Time-of-Flight (ToF) measurement. At $110 \mathrm{~K}$, the NDM effect was most clearly pronounced. ${ }^{10}$ An analytic expression of how the magnitude of electron drift velocity $\left(v_{d}\right)$ depends on the magnitude of the electric field $(E)$ is found by fitting our experimental electron mobility data at $110 \mathrm{~K}$ (seen in Fig. 2(b)) to the quotient of secondorder polynomials. The analytic expression

$$
\begin{aligned}
v_{d} & =\mu_{n}(E) \times E \\
& =\frac{\left(1.29 E^{2}-349 E+2.55 \times 10^{5}\right) \times 10^{3}}{E^{2}-385 E+6.92 \times 10^{4}} \times E,
\end{aligned}
$$

with the electron drift velocity given in $\mathrm{cm} / \mathrm{s}$, and the electric field given in $\mathrm{V} / \mathrm{cm}$ describes well the experimental data for $E \leq 1400 \mathrm{~V} / \mathrm{cm}$. This means that the electron mobility $\mu_{n}$ (in contrast to the hole mobility, $\mu_{p}$ ) is not assumed constant. Instead it is strongly electric field dependent as can be seen from the expression above.

The proposed experiment is based on our previous results ${ }^{10}$ that were achieved through $\mathrm{ToF}$ measurements where a pulsed UV laser was used to generate electron-hole pairs. The inset in Fig. 2(b) shows a plot with data from a ToF experiment together with the results from a driftdiffusion simulation, showing good agreement for the measured current. This comparison was done to give credibility to the use of drift-diffusion transport simulations and to the choice of contact model. Here, we assume blocking contacts and these are modeled using contact recombination
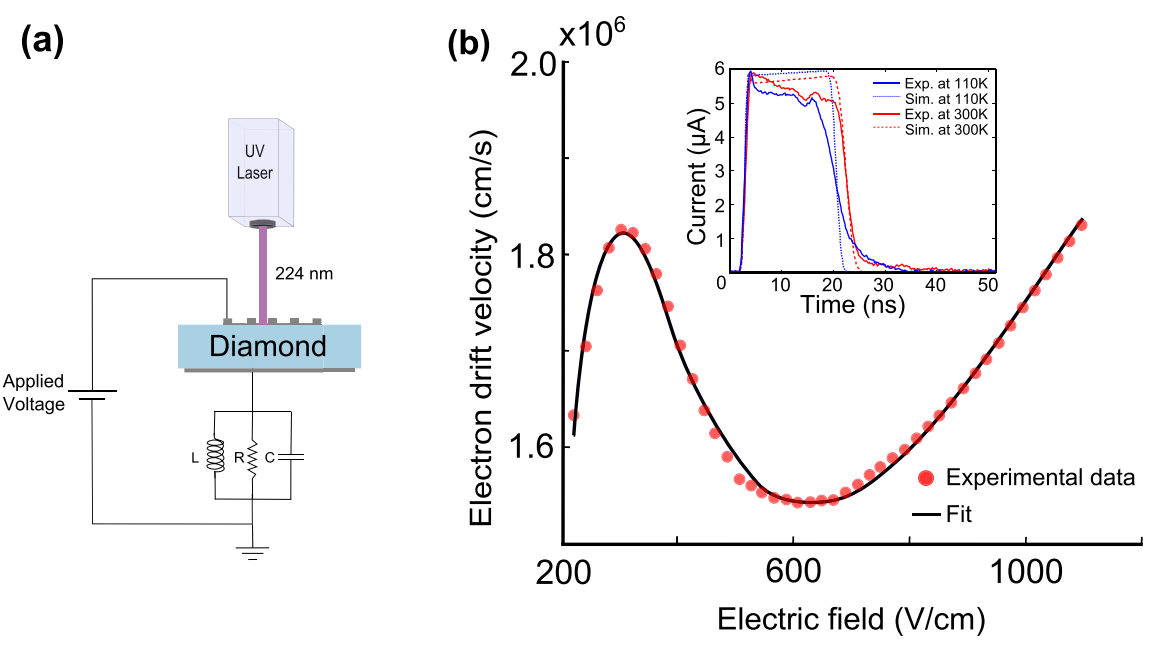

FIG. 2. (a) Schematic of the TEO used in the simulations, with an RLC circuit as a resonator. (b) Fitting an expression to the experimental drift velocity observation within the NDM region at $110 \mathrm{~K}$. The inset in (b) shows a comparison between drift-diffusion simulations and ToF measurements performed at $110 \mathrm{~K}$ and $300 \mathrm{~K}$ with an applied voltage of $30 \mathrm{~V}$. 

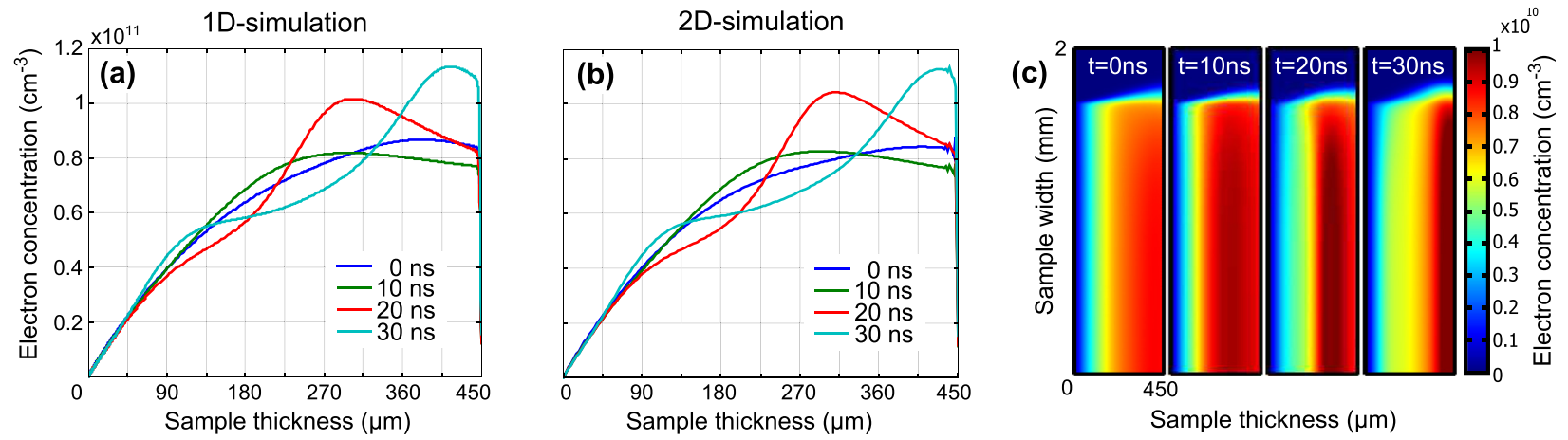

FIG. 3. Change of electron concentration within the sample for half of a period at the steady-state of the oscillation ( $>2 \mu$ s) for both the 1D and 2D simulations. (a) Results of the 1D simulation with a plot of the electron concentration at each position in the sample at different times and (b) results of the 2D simulation. (c) The electron concentration achieved in the $2 \mathrm{D}$ simulation at different times. This figure shows that at $10 \mathrm{~ns}$, the electron cloud starts to grow bigger and at $30 \mathrm{~ns}$ the electrons are extracted at the contact.
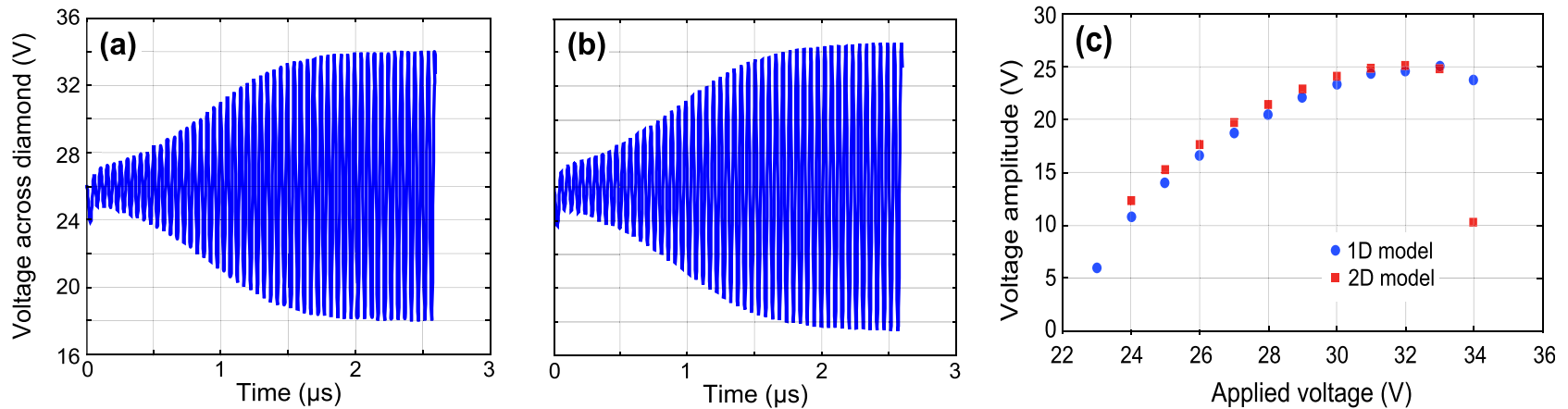

FIG. 4. (a) and (b) show simulation results of the voltage across the diamond as a function of time for $450 \mu \mathrm{m}$ thick sample at $\mathrm{T}=110 \mathrm{~K}$ with an applied voltage of $26 \mathrm{~V}, \mathrm{~L}=33 \mu \mathrm{H}, \mathrm{C}=1.05 \mathrm{pF}$, and $\mathrm{R}=12.3 \mathrm{M} \Omega$ for $1 \mathrm{D}$ an $2 \mathrm{D}$ respectively. (c) comparison of the amplitude between the $1 \mathrm{D}$ and $2 \mathrm{D}$ model as a function of applied voltage. The models reveal similar trend and results.

velocity $v_{\text {recomb. }}>10^{6} \mathrm{~cm} / \mathrm{s}$. These are the types of contacts routinely used in ToF measurements.

For the simulation of TEO, we use a continuous carrier generation with just-above-bandgap photons having a deep penetration depth that are absorbed according to the Beer-Lambert law and can be expressed as

$$
g_{n}=g_{p}=I \kappa e^{-\kappa x}
$$

where $I$ is the photon flux, $\kappa$ the absorption coefficient, and $x$ is the penetration depth. For deep penetration, a possible source would be a HeAg-excimer laser at a wavelength of $224 \mathrm{~nm}$. Commercial quasi-CW HeAg lasers are becoming available in the market, with pulse lengths in the $100 \mu$ s range, i.e., much longer than the electron transient time (ns). Constants used in the model are $I=1.776 \times 10^{17} \mathrm{~cm}^{-2} \cdot \mathrm{s}^{-1}$, $\kappa=50 \mathrm{~cm}^{-1}, \quad \varepsilon_{r}=5.7, \quad T=110 \mathrm{~K}, \quad$ and $\quad E_{g}=5.47 \mathrm{eV}$. Additionally, $\tau_{n}=\tau_{p}=10 \mu$ s was used in the simulations. ${ }^{12}$ However, any values of $\tau_{n}$ and $\tau_{p}$ above 100 ns yield essentially the same results. The hole mobility is assumed to be $12000 \mathrm{~cm}^{2} / \mathrm{V} \mathrm{s}$ and field independent. ${ }^{17}$ The diamond is connected in series to a resonant circuit, the total current through the TED is equal to the total current through the TEO, and the voltage over the TED is the difference of the applied voltage and the voltage across the resonant circuit, see Fig. 2(a).

Electron-hole pairs are generated in the bulk with the highest generation rate near the illuminated contact and gradually diminishing towards the other contact as the light is attenuated in passing through the diamond. Because of the polarity of the applied voltage, holes will be extracted at the illuminated side while electrons will drift to the other side. In 1D, we assume one-dimensional diffusion along the E-field direction. In 2D, we assume diffusion in the plane. The influence of diffusion is rather small and can be neglected, but we have chosen to include it in the simulations for completeness. If the voltage over the TED is such that the associated electric field in the absence of charge is within the negative resistance region, an excess in the electron concentration will tend to grow with time until the electrons are extracted at the contact, as can be seen in Fig. 3. The figure shows the 1D and 2D model simulation results of the electron concentration for half of the oscillation period for steady-state oscillations.

By generating free charge carriers with a continuous light source, the process explained above builds up electric current instabilities and gives rise to oscillations. This can be

TABLE I. Parameters varied in the 1D simulations.

\begin{tabular}{lccccc}
\hline \hline & & \multicolumn{3}{c}{ In RLC circuit } \\
\cline { 4 - 6 } Sweep & $\begin{array}{c}\text { Voltage } \\
\text { No. }\end{array}$ & $\begin{array}{c}\text { Sample } \\
\text { thickness }(\mu \mathrm{m})\end{array}$ & $\begin{array}{c}\text { Inductance } \\
(\mu \mathrm{H})\end{array}$ & $\begin{array}{c}\text { Capacitance } \\
(\mathrm{pF})\end{array}$ & $\begin{array}{c}\text { Resistance } \\
(\mathrm{M} \Omega)\end{array}$ \\
\hline 1 & $15-35$ & 450 & 33 & 1.05 & 12.3 \\
2 & 31 & 450 & $25-150$ & 1.05 & 12.3 \\
3 & 31 & 450 & 85 & $0.5-5$ & 12.3 \\
4 & 31 & 450 & 85 & 1.05 & $0.05-60$ \\
5 & 31 & $50-600$ & 85 & 1.05 & 12.3 \\
\hline \hline
\end{tabular}



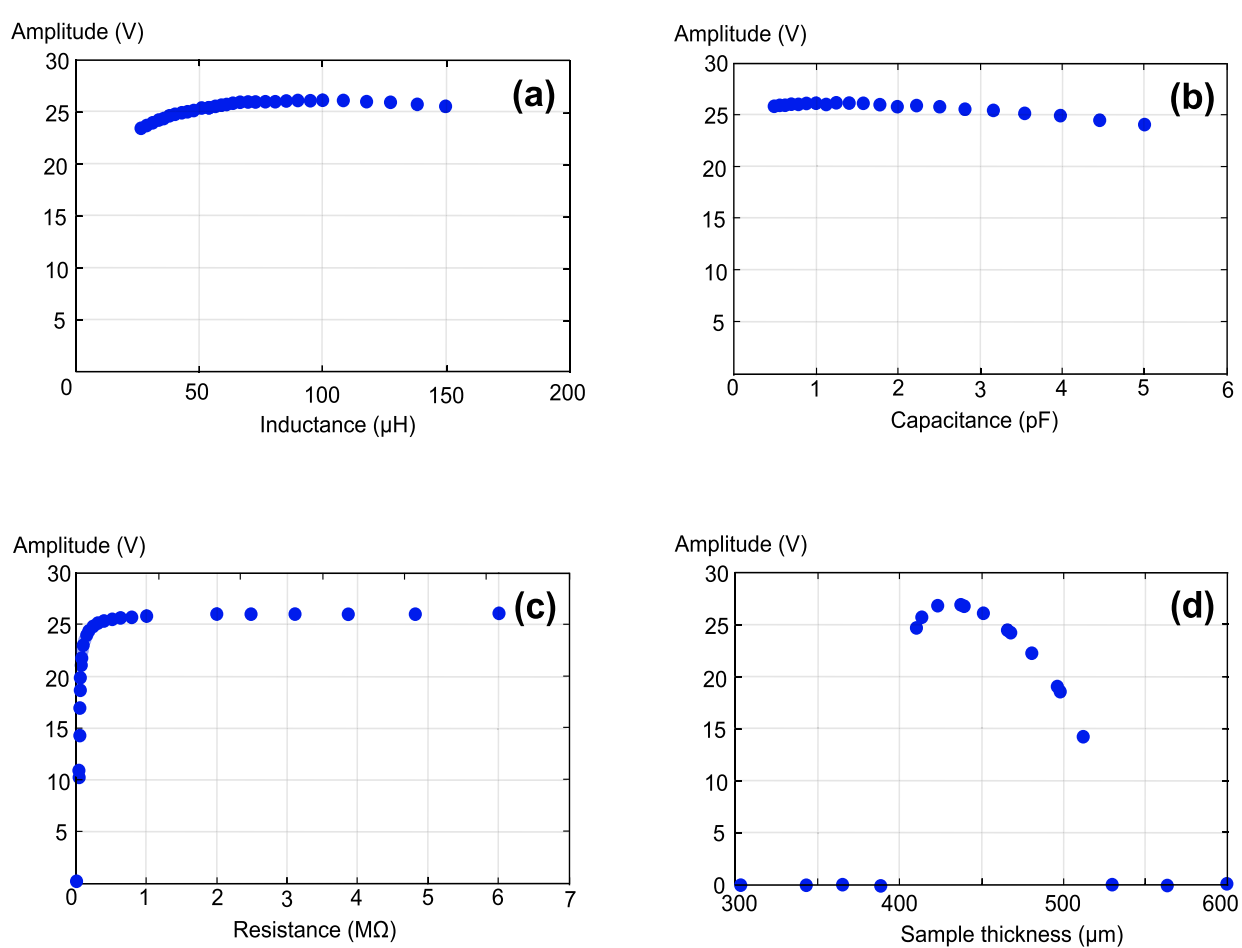

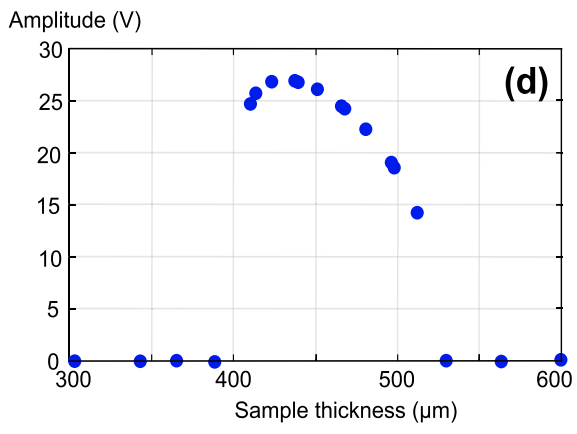

FIG. 5. Visualization of the results of the simulations, where in (a), the inductance is varied; in (b), the capacitance is varied; in (c), the resistance in the RLC circuit is varied; and in (d), the sample thickness is varied. observed as changes of voltage across and changes of current through the diamond over time. The results of these voltage oscillations as a function of time for both the 1D and 2D model can be seen in Fig. 4. They show the desired gradual increase in oscillation amplitude (defined as the difference between the maximum and minimum voltage), which eventually reaches a periodic steady-state. The rather good agreement between the 1D and 2D model indicates that there are no significant advantages of using the 2D model. Because of this and due to the increased simulation time using the $2 \mathrm{D}$ model, further investigations focus on the 1D simulation of the TEO. The sample thickness, applied voltage, inductance, capacitance, and resistance of the RLC circuit are varied to optimize the oscillations in the device, and the values of these parameters are summarized in Table I.

The influence of varying the parameters can be seen in Fig. 5. The transferred-electron oscillations occur at an applied voltage of $25-34 \mathrm{~V}$ for a sample with thickness in the range of $400-500 \mu \mathrm{m}$. The exact values of inductance and capacitance in the circuit have no critical influence on the amplitude, but the resistance should be kept above $0.5 \mathrm{M} \Omega$ for oscillations to occur. In addition, previous results indicate that the diamond sample has to have a neutral impurity concentration lower than $2 \times 10^{15} \mathrm{~cm}^{-3}$ and an ionized impurity concentration lower than $4 \times 10^{13} \mathrm{~cm}^{-3}$ in order to achieve the NDM. ${ }^{10}$

Previous experiments have demonstrated the presence of NDM in diamond. This follow-up paper exploits this property and investigates the possibility of achieving transferred-electron oscillations, using FEM simulations. After comparing the 1D and 2D models, one could establish the absence of significant discrepancy between these models; hence, the focus is on the 1D simulations. According to the presented findings, it is possible to create such oscillations in diamond devices at around $110 \mathrm{~K}$. It seems that the applied voltage and sample thickness are more critical than the parameters of the resonator. These discoveries are highly encouraging concerning the prospect of fabricating TEO devices based on diamond and give a detailed insight on how to achieve this goal. The experimental discovery of transferred-electron oscillation in diamond could lead to important devices in microwave applications such as microwave generators or power amplifiers.

The authors would like to thank the Swedish Research Council, Grant No. 621-2012-5819. S.M. wishes to thank the Royal Swedish Academy of Science (KVA) and the Japan Society for the Promotion of Science (JSPS) for their financial support. M.G. wishes to thank the ÅForsk Foundation, Grant No. 15-288.

${ }^{1}$ J. B. Gunn, Solid State Commun. 1, 88 (1963).

${ }^{2}$ J. B. Gunn, IEEE Trans. Electron Devices 23, 705 (1976).

${ }^{3}$ H. Kroemer, Proc. IEEE 52, 1736 (1964).

${ }^{4}$ J. W. Allen, M. Shyam, and G. L. Pearson, Appl. Phys. Lett. 9, 39-42 (1966).

${ }^{5}$ G. W. Ludwig, R. E. Halsted, and M. Even, IEEE Trans. Electron Devices ED-13, 671 (1966).

${ }^{6}$ M. R. Oliver and A. G. Foyt, IEEE Trans. Electron Devices 14, 617 (1967).

${ }^{7}$ G. W. Ludwig, IEEE Trans. Electron Devices 14, 547 (1967).

${ }^{8}$ T. K. Ishii, Components and Devices (Academic Press, 1995), Vol. 1.

${ }^{9}$ C. Canali, A. Loria, F. Nava, and G. Ottaviani, Solid State Commun. 12, 1017 (1973).

${ }^{10}$ J. Isberg, M. Gabrysch, S. Majdi, and D. J. Twitchen, Appl. Phys. Lett. 100, 172103 (2012).

${ }^{11}$ J. Isberg, M. Gabrysch, J. Hammersberg, S. Majdi, K. K. Kovi, and D. J. Twitchen, Nat. Mater. 12, 760 (2013).

${ }^{12}$ J. Hammersberg, S. Majdi, K. K. Kovi, N. Suntornwipat, M. Gabrysch, D. J. Twitchen, and J. Isberg, Appl. Phys. Lett. 104, 232105 (2014).

${ }^{13}$ H. Löfas, A. Grigoriev, J. Isberg, and R. Ahuja, AIP Adv. 1, 032139 (2011).

${ }^{14}$ P. Pavone, K. Karch, O. Schutt, W. Windl, D. Strauch, P. Giannozzi, and S. Baroni, Phys. Rev. B 48, 3156 (1993).

${ }^{15}$ K. K. Ng, Complete Guide to Semiconductor Devices, 2nd ed. (John Wiley \& Sons, Inc., 2002), pp. 64-74.

${ }^{16}$ S. M. Sze and M.-K. Lee, Semiconductor Devices: Physics and Technology, 3rd ed. (John Wiley \& Sons, Inc., 2012).

${ }^{17}$ M. Gabrysch, S. Majdi, D. J. Twitchen, and J. Isberg, J. Appl. Phys. 109, 063719 (2011). 